\title{
Structured control from self-organizing arm movements
} Katja Fiedler*1, Georg Martius ${ }^{1,2}$, Frank Hesse ${ }^{1,2}$ and J Michael Herrmann 1,2,3

\author{
Address: ${ }^{1} \mathrm{MPI}$ for Dynamics and Self-Organization, Goettingen, Bunsenstr. 10, 37073 Goettingen, Germany, ${ }^{2}$ Bernstein Center for Computational \\ Neuroscience Goettingen, Bunsenstr. 10, 37073 Goettingen, Germany and ${ }^{3}$ Inst. of Perception, Action and Behaviour, School of Informatics, \\ University of Edinburgh, EH9 3JZ, UK \\ Email: J Michael Herrmann - michael.herrmann@ed.ac.uk \\ * Corresponding author
}

from Seventeenth Annual Computational Neuroscience Meeting: CNS*2008 Portland, OR, USA. 19-24 July 2008

Published: II July 2008

BMC Neuroscience 2008, 9(Suppl I):P74 doi: I0.1 I86/I47I-2202-9-SI-P74

This abstract is available from: http://www.biomedcentral.com/I47I-2202/9/SI/P74

(c) 2008 Fiedler et al; licensee BioMed Central Ltd.

\section{Introduction}

The organization of unconstrained arm movements in humans appears to be determined essentially by the biophysical properties of the limb [1], which, however, might imply as well that the underlying neural control mechanisms are perfectly adapted to controlled system. The stiffness of the arm with respect to perturbing forces [2] is caused by an active process that cannot be inferred from the biomechanics of the arm alone, and may thus reveal information about the underlying neural control mechanisms. We approach the problem by analyzing experimental measurements of stiffness in human subjects and by simulations of emergent control of a model of the human arm.

\section{Physical simulations of a controlled human arm} Control is achieved by an algorithm for self-organizing control of sensorimotor loops [3] that establishes a dynamical system in the sensor space. The resulting dynamics is characterized by both the sensitive dependence on previous actions as well as the local predictability by an internal model. The algorithm merely follows the objective of conveying the sensorimotor loop into a marginally stable regime, which has been shown to suffice to generate a variety of behaviors in different robots [3]. Here we study the example of a four DOF model of a human arm. The controller creates a manifold of behaviors by an itinerant motion across the critical region of its parameter space. In order to identify "natural" behaviors a set of controllers is used. An effective controller can be selected by maximal learning progress [4] and upon convergence to a local optimum the active controller is stored as elemen- tary behaviors in a premotor layer and can be reactivated by environmental affordances or by high-level planning.

\section{Experiments on arm movements in human subjects}

In parallel we have studied properties of human arm movements in a four-DOF setting (wrist-restrained) in three spatial dimensions using a high-performance haptic device. We have measured the stiffness based on the equilibrium point hypothesis by the motor response to a perturbing force and in dependence on the spatial position of the arm. In a second set of experiments we have studied trajectories in a self-referential task, where the subjects chase a target which is driven by a fast-learning controller. The controller learns to predict the subject's movement such that an increasing difficulty of the task is achieved and more complex movements can be expected.

\section{Results}

While at low complexity in the chasing task closed-loop control is sufficient, the subjects become more likely to use preexisting open-loop movement primitives at an increased task complexity. The primitives are extracted from the data by third-order statistics and share geometrical properties with the primitives that are produced by self-organizing multi-agent control of the simulated arm. The measurement of the stiffness allows us to derive a common explanation for the control-related aspects of the movement primitives in both experiment and simulation. Furthermore, the spatial heterogeneity of the extent and density of the primitives can be related to the stiffness estimates. Finally, we discuss application to prosthetics and 
suggest as a principle for the organization of the working range of arm movements that the entire sensorimotor space is partitioned into segments where the associated elementary behavior assumes optimal controllability.

\section{Acknowledgements}

The authors thank J. Schroeder-Schetelig for assistance. K.F. has received support by the "Compositionality" project (DIP-FI.2), G.M. and F.H. have received support by BCCNGoe grant \#0IGQ0432

\section{References}

I. Biess A, Liebermann DG, Flash T: A computational model for redundant human 3D pointing movements. Journal of Neuroscience 2007, 27(48): I3045-13064.

2. Flash T, Mussa-Ivaldi F: Human arm stiffness characteristics during the maintenance of posture. Experimental Brain Research 1990, 82:315-326.

3. . http://robot.informatik.uni-leipzig.de/research/publications, http:// robot.informatik.uni-leipzig.de/research/videos

4. Herrmann JM: Dynamical Systems for Predictive Control of Autonomous Robots. Theory in Biosciences 2001, 120(34):24I-252.

Publish with Bio Med Central and every scientist can read your work free of charge

"BioMed Central will be the most significant development for disseminating the results of biomedical research in our lifetime. "

Sir Paul Nurse, Cancer Research UK

Your research papers will be:

- available free of charge to the entire biomedical community

- peer reviewed and published immediately upon acceptance

- cited in PubMed and archived on PubMed Central

- yours - you keep the copyright

Submit your manuscript here:

http://www.biomedcentral.com/info/publishing_adv.asp 